\title{
The Potential Role of MT and Vimentin Immunoreactivity in the Remodeling of the Microenvironment of Parotid Adenocarcinoma
}

\author{
Magdalena Dutsch-Wicherek • Agata Lazar • \\ Romana Tomaszewska
}

Received: 17 October 2010 /Accepted: 25 November 2010 / Published online: 15 December 2010

(C) The Author(s) 2010. This article is published with open access at Springerlink.com

\begin{abstract}
A tumor stimulates the remodeling of its microenvironment in order to control and accelerate its own growth and to initiate metastases. To create metastases the tumor cells must first acquire the ability to detach from the main tumor and to adhere to, invade, and degrade the adjacent extracellular matrix. The cells must then be able to enter the lumen of the vessels where they home the distant tissues and organs by forming secondary tumors. The acquisition of this phenotype is related to the phenomenon of epithelial-to-mesenchymal transition. On the molecular level, this process is typified by a change in the expression of epithelial markers and by the enhancement of the expression of mesenchymal markers like vimentin that are responsible for cell migration and invasion. Metallothioneins have been shown to help protect against apoptosis. The expression of MT by tumor cells plays an important and complex role not only because of its pro-proliferative, anti-apoptotic activity, but also because it inhibits the immune response. The aim of the present study was to evaluate the immunoreactivity of vimentin and MT in the salivary gland adenocarcinoma and its stroma in order to observe the phenomenon of stromal remodeling. The tissue samples of salivary gland adenocarcinomas and their stromas and the palatine tonsils which constituted the reference group were obtained during routine surgical procedures. The immu-
\end{abstract}

\footnotetext{
M. Dutsch-Wicherek $(\triangle)$

Otolaryngology Head and Neck Surgery Department, Jagiellonian University,

Krakow, Poland

e-mail: mowicher@gmail.com
}

A. Lazar $\cdot$ R. Tomaszewska

Pathology Department, Jagiellonian University,

Krakow, Poland noreactivity of vimentin, metalothionein, CD56, CD57 antigens was evaluated by the immunohistochemistry method in 30 tissue samples of parotid adenocarcinoma. The patient's consent was obtained in each case. A statistically significantly higher level of MT immunoreactivity was observed in the adenocarcinoma tissue slides than in either the stromal slides or the reference slides while no differences in MT immunoreactivity were detected when the stroma and reference tissue slides were compared. A statistically significantly higher vimentin immunoreactivity level was identified in the tumor microenvironment tissue slides than in the tumor tissue slides, and a statistically significantly higher level of vimentin immunoreactivity was identified in the tumor microenvironment slides than in the slides of the reference tissue, while no differences were identified between the adenocarcinoma tissue slides and the reference slides with respect to vimentin immunoreactivity. A statistically significantly higher number of CD56- and CD57expressing cells were identified in the reference tissue slides than in either the adenocarcinoma or stromal slides. In conclusion, the stroma of salivary gland adenocarcinoma in this study has been characterized by remodeling. The remodeling is represented by the expression of both vimentin and MT and by a deficit of CD57- and CD58expressing cell infiltration. This situation would seem to be the result of immune tolerance for the tumor developing within the tumor microenvironment. Furthermore, the presence of MT and vimentin immunoreactivity in the fibroblasts of the tumor stroma may constitute a marker of active tissue remodeling.

Keywords Tumor microenvironment .

Carcinoma-associated fibroblasts - Metallothionein .

Vimentin - Epithelial to mesenchymal transition 


\section{Introduction}

A tumor stimulates the remodeling of its microenvironment in order to control and accelerate its own growth and to initiate metastases. The tumor thus induces angiogenesis and forms new capillaries of enhanced permeability, changes the structure of the extracellular matrix and the function of the cells (including the immune system cells), and activates the homing of the tumor microenvironment by the immune cells (which with their altered function become responsible for the chronic inflammation that accompanies tumor development as compared with a non-healing wound) [1-5]. The tumor microenvironment is composed of cells, extracellular matrix, and proteins. Within the microenvironment, the endothelial cells, tumor-associated macrophages, and carcinomaassociated fibroblasts have been identified as the most important for promoting tumor progression [1-5].

To create metastases the tumor cells must first acquire the ability to detach from the main tumor and to adhere to, invade, and degrade the adjacent extracellular matrix. The cells must then be able to enter the lumen of the vessels where they home the distant tissues and organs by forming secondary tumors. The acquisition of this phenotype is related to the phenomenon of epithelial-to-mesenchymal transition (EMT) [6-8]. On the molecular level, this process is typified by a change in the expression of epithelial markers and the enhancement of the expression of mesenchymal markers responsible for cell migration and invasion. EMT is a physiological process observed during embryological development and the remodeling of tissue during wound healing and chronic inflammation [9]. The metastasizing cells demonstrate features similar to cells undergoing EMT, and EMT is thought to participate in tumor progression. EMT is defined as the phenomenon which occurs when the cell loses its stable, polarized, nonmigrational abilities and transforms into a mesenchymal cell with fibroblastic, migrational features. As the cells lose their polarization, a change in both the cell-to-cell and cellto-extra-cellular matrix adhesion occurs, leading to higher cell mobility [10]. During EMT, epithelial cells progressively redistribute and decrease the expression of the proteins typical for the apex and basis of the cell, such as cell connection proteins which include E-cadherine. In turn, mesenchymal proteins such as vimentin and $\mathrm{N}$-cadherine are re-expressed by the cells. These changes lead to the disappearance of the connections between the cells and as a result the cells acquire the mobility needed to invade the tissues [11].The mesenchymal phenotype can also be reversed by blocking vimentin expression; this results in the re-expression of epithelial features and a decrease in tumor aggressiveness [12]. Since vimentin has never been studied in salivary gland adenocarcinomas we aimed to determine its immunoreactivity in these tumors and their microenvironments in order to observe the remodeling of the tumor microenvironment. Vimentin is one of the markers for identifying activated fibroblasts or the myofibroblasts of the tumor microenvironment. These are also called carcinoma-associated fibroblasts $(\mathrm{CAF})$ as the number of these cells increases significantly in various malignant tumors. They constitute an important component of the tumor microenvironment and participate in the tumor's development [13]. Myofibroblasts have many features in common with both smooth muscle cells and fibroblasts and induce the growth and differentiation of cells during embryogenesis, wound healing, and other physiological processes related to tissue remodeling [13]. The most common precursor cells for myofibroblasts are fibroblasts, but the smooth muscles of the vessels, pericytes, precursor bone marrow cells, and even cancer cells can transdifferentiate into myofibroblasts. PDGF secreted by the tumor stimulates fibroblast proliferation; TGF- $\beta$ secreted by macrophages chemoattracts fibroblasts in lower concentration, while in higher concentration it induces the transdifferentiation into myofibroblasts [13]. Myofibroblasts appear shortly before tumor invasion and degrade the basal membrane and extracellular matrix by secreting serine proteases and matrix metalloproteinases. The stromal fibroblasts in oral squamous cell carcinomas have been demonstrated to be associated in a significant way with carcinomas but not with pre-malignant lesions [14]. Myofibroblasts express insulin-like and hepatocyte growth factors which induce and stimulate the survival and migration of cells as well as the expression of proangiogenic and pro-inflammatory factors. Myofibroblasts stimulate not only their own migration to the tumor, but also the survival, proliferation, and invasion of tumor cells and the process of angiogenesis. Together these factors increase the ability of the tumor to grow and disseminate [15-17]. CAF are routinely identified by such markers as: alpha-smooth muscle actin (alpha-SMA), vimentin, S100A4 protein/fibroblast specific protein-1 (FSP1), and type-I collagen [18].

Tumor microenvironment remodeling may also be characterized by changes in MT expression. Metallothioneins (MTs) have been shown to play a protective role against apoptosis [19] and may also be important for the proliferation and differentiation of cells [20, 21]. MT expression in the cytoplasm of the cell has been found to be involved in protecting against cytotoxicity while the nuclear expression of MT is involved in protecting against genotoxicity. Genotoxicity involves the acquisition of the malignant phenotype by the cell; this is associated with the specific mutations that play key roles in carcinogenesis [20-23]. Cytotoxicity, by contrast, is involved in the interactions of immune system cells with cancer cells. The role played by the expression of MT by tumor cells is important and 
complex, not only because of the pro-proliferative, antiapoptotic activity of this protein, but also because it inhibits the immune response [24-27].

The tumor controls the immune response in the tumor microenvironment and modifies the antitumor immune response. There are two populations of lymphocytes or NKTs (natural killer-like T cells), CD56-expressing cells and CD57expressing cells. These cells are typified by an immunoregulatory role $[28,29]$. CD56-positive $\mathrm{T}$ lymphocytes demonstrate an effector function of cytotoxic T cells [30], while CD57-positive $\mathrm{T}$ lymphocytes demonstrate an immunoregulatory role for $\mathrm{T}$ cytotoxic lymphocytes. The expression of CD57 antigen is also related to $\mathrm{T}_{\mathrm{EM}}$ (effector memory T cells), especially a significant number of $\mathrm{T}_{\mathrm{EMRA}}$ (expressing CD45RA CD ${ }^{+} \mathrm{CD}^{+} \mathrm{CD} 57+\mathrm{CD} 28$ - and high perforin expression) [31,32]. NKT $\mathrm{T}_{\mathrm{EM}}$ cells are typified by an immediate effector function [32]. As the immunohistochemistry method allows us to identify only one antigen at a time, in the current study we were not able to identify the type of cell precisely. We have therefore determined only the immunoreactivity levels of CD56 and CD57 antigens in salivary gland adenocarcinomas and their microenvironments.

\section{Materials and Methods}

\section{The Group of Patients}

\section{Patients with Salivary Adenocarcinoma}

All the tissue samples were derived from patients whose salivary gland tumors were surgically removed in the Otolaryngology Department of the Jagiellonian University. The patient's consent was obtained in each case. Additionally, approval for the research program was granted from the Ethical Committee of the Jagiellonian University in Krakow: KBET/90/B/2005. We recruited 30 patients to our study from those who had undergone the surgery between January 2000 and December 2008. All the tumors originated from parotid glands; tumors originating from other smaller salivary glands were excluded from the present study. In each case parotidectomy along with lymphadenectomy was performed. The tumors were removed in healthy surgical margins. The presence of lymph node metastases in these removed lymph nodes was then evaluated. All the patients were treated surgically, and none of the patients enrolled in the study had been pre-treated using chemo- or radiotherapy. Additionally, we have compared the immunoreactivity of analyzed antigens in both primary and recurrent tumors, but were unable to identify any significant associations.

All the tissue samples were histopathologically verified. Pathological analysis using the classical hematoxylin and eosin staining techniques after fixation in a formalin of the surgically removed material was performed in the Pathology Department of the Jagiellonian University by two experienced pathologists (R.T. and A.L.) working independently. The tissue material was then fixed in the solution of $10 \%$ formalin, rinsed, dehydrated, and transferred through a progressively increasing concentration of ethanol (from $50 \%$ to absolute alcohol); it was passed through xylens I, II, and III as well as molten paraffin wax. Lastly, the tissue blocks were sectioned and placed onto 3-4 $\mu \mathrm{m}$ slides. The process was mainly automated, but both the paraffin embedding and the sectioning of the tissue samples into 3-4 $\mathrm{mm}$ slides were performed manually.

The clinical characteristics of the subjects are presented in Tables 1 and 2.

\section{Reference Group}

As a reference group we chose palatine tonsils that had been removed due to recurrent tonsillitis. In these tissue samples we evaluated the epithelium lining the tonsils, and not the lymphoid tissue (Table 2).

Table 1 The clinical characteristics of the patients with salivary adenocarcinomas

\begin{tabular}{lr}
\hline The number of patients & 30 \\
$\mathrm{M}^{\mathrm{a}}$ & 13 \\
$\mathrm{~W}^{\mathrm{a}}$ & 17 \\
Age range (average) & \\
Tumor stage & 25 \\
I-II & 5 \\
III-IV & \\
The presence of lymph node metastases & 25 \\
N0 & 5 \\
N+ & \\
Tumor size & 29 \\
T1-T2 & 1 \\
T3-T4 & 1 \\
Tumor grade & 22 \\
G1 & 7 \\
G2 & \\
G3 & \\
Histological types of tumors & 16 \\
Adenocarcinoma & 14 \\
Adenocarcinoma acinocellulare & \\
Tumors & \\
Primary & \\
Recurrence & \\
\hline
\end{tabular}

${ }^{\text {a }} M$ men, $W$ women

${ }^{\mathrm{b}}$ Tumor stage was evaluated according to TNM classification 6th edition, 2002 
Table 2 The characterization of the reference group

\section{Palatine tonsils}

The number of patients

$\mathrm{M}^{\mathrm{a}}$

$\mathrm{W}^{\mathrm{a}}$

Age range average

$\mathrm{M}^{\mathrm{a}}$

$\mathrm{W}^{\mathrm{a}}$

${ }^{\mathrm{a}} M$ men, $W$ women

\section{Immunohistochemical Analysis}

In the present study we aimed to analyze the immunoreactivity of various antigens in parotid adenocarcinoma and its stroma; we were also interested in the distribution of the antigen immunoreactivity among the whole tissue of the tumor, including the tumor stroma. For this reason we chose the immunohistochemistry method for the present study. This is the only method that shows the actual architecture of the dialogue between the tumor and its stroma.

From every tumor 3-4 tissue samples were taken (depending on the tumor size; if, for instance, the tumor was $1 \mathrm{~cm}$ in diameter, then it was subject to further analysis). Every tissue sample was embedded in paraffin and formed into a tissue block which was then cut into tissue slides. All the tissue slides were further histopathologically verified and for the additional analysis the most representative slides were selected for immunohistochemistry (for the analysis of the tumor samples and the stromal samples and in order to assess the tumor-stroma interaction).

Two observers, working independently and having no knowledge of the clinicopathological data, reviewed the immunohistochemical expression of MT, vimentin, CD56and CD57-positive cells. Immunohistochemical analysis was performed in the Pathology Department of the Jagiellonian University. Five-micrometer slides from each case were deparaffinized, rehydrated, and rinsed in distilled water. Endogenous peroxidase activity was blocked by 8 min of incubation in $3 \% \mathrm{H} 2 \mathrm{O} 2$ at room temperature. The slides were then rinsed and immersed in boiling citrate buffer ( $\mathrm{pH}$ 6.0) in a microwave oven with three changes of buffer for $5 \mathrm{~min}$ each. Immunohistochemistry was performed in each case applying the Envision method using Dako Autostainer. The samples were stained automatically. The immunohistochemical staining was based on antigenantibody reaction. The microscopy was performed using an Axio Zeiss microscope and the tissue slides were evaluated under both 20x and 40x magnification.

The following antibodies were applied: CD56, CD57 (Novocastra in dilution 1:50), Vimentin (DAKO, Denmark, in dilution 1:50), and Metallothionein (ABCAM, in dilution $1: 25$ ) for $10 \mathrm{~min}$ at room temperature. Sections were counterstained with hematoxylin and mounted in glycergel. As a positive control, a tonsil specimen was taken for Metallothionein. All stainings were performed with the same procedure only the primary antibody was omitted as a negative control. Metallothionein expression was evaluated in entire slides in the area of the tumor and in the stroma, as
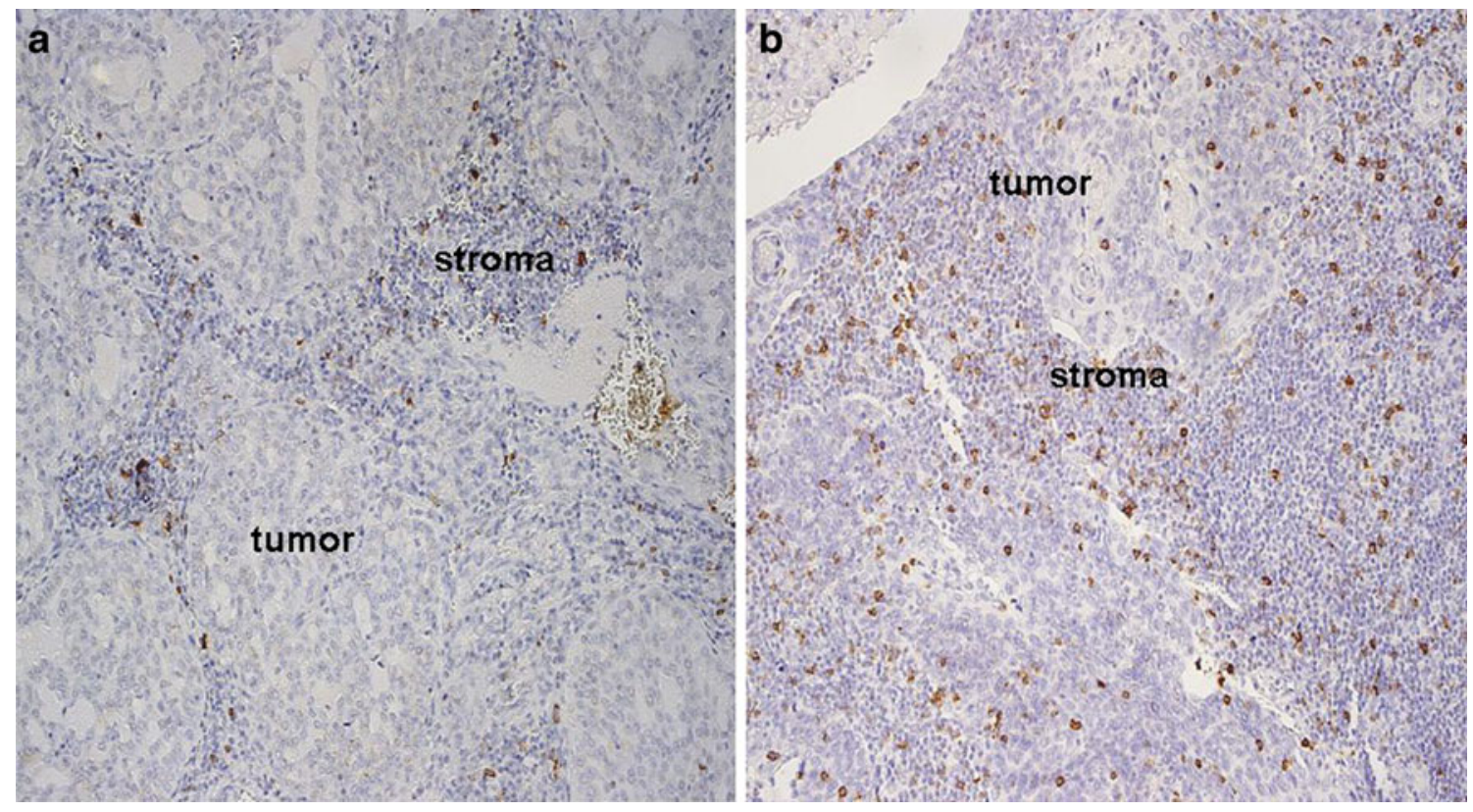

Fig. 1 The immunoreactivity of CD56 (A) and CD57 (B) antigens in adenocarcinoma and its stroma, representing the cytoplasmic pattern of staining. Moderately differentiated adenocarcinoma; sample magnification 20x 
follows: 0 -no reactivity; +1 -weak, when observed any cytoplasmic staining pattern (also granular in the paranuclear region) in up to $10 \%$ of positive cells; +2 -marked cytoplasmic (sometimes together with membranous) staining in $11-30 \%$ of the cells); +3 - high expression (more than $30 \%$ of positive cells). Similarly, vimentin expression was evaluated in the entire slides both in the area of the tumor and in the stroma, as follows: 0 -no reactivity; +1 - weak, when observed any (also granular in paranuclear region) cytoplasmic staining pattern (in up to $10 \%$ of positive cells); $+2-$ marked cytoplasmic (sometimes together with membranous) staining in $11-30 \%$ of the cells); +3 - high expression (more than $30 \%$ of positive cells). Variable scales were used to evaluate an amount of the cells semi-quantitatively, depending on their general number in the specimen, an average cell number per $1 \mathrm{hpf}$ (high power field, objective magnification $\mathrm{x} 40$ ). CD56+ and CD57+ cells were thus estimated as follows: 0 - lack of positive cells; +1 - single positive cells in the specimen; $+2-2-5$ positive cells per $1 \mathrm{hpf} ;+3$-more than 5 positive cells $/ 1 \mathrm{hpf}$.

\section{Statistical Analysis}

The distribution of variables in the study groups of the patients checked with the use of the Shapiro-Wilk test showed that each of the patients was in fact different from normal. The statistical significance between the groups was determined by the Kruskal-Wallis test, oneway analysis of variance by ranks. The Mann-Whitney $U$ test was then used as applicable. All statistical analyses were carried out with the Statistica 8.0 software program. A $p$ value $<0.05$ was considered indicative of statistical significance.

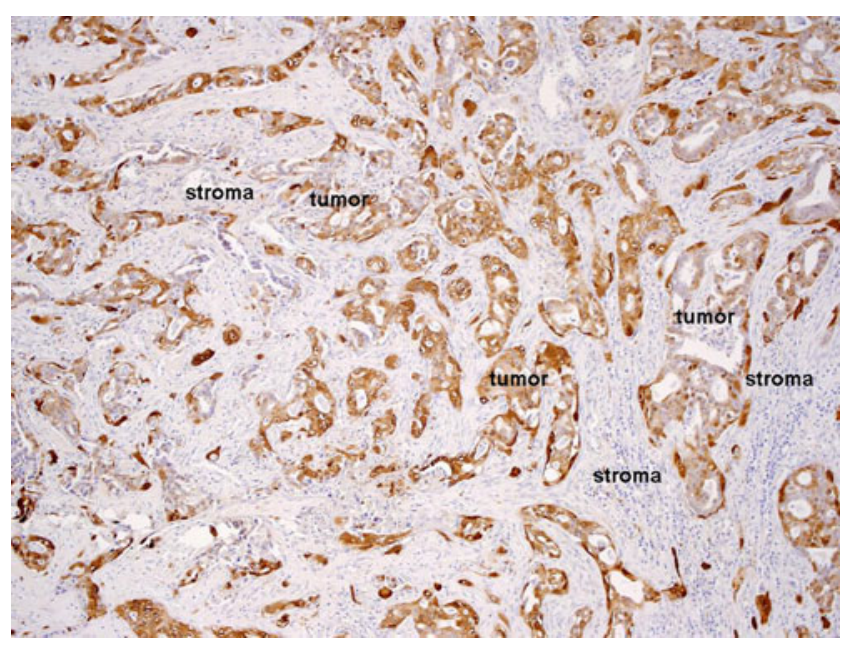

Fig. 2 MT antigen immunoreactivity in adenocarcinoma and stromal tissue slides. The MT immunoreactivity in adenocarcinoma represents the nuclear-cytoplasmic pattern of staining. Moderately differentiated adenocarcinoma; sample magnification 20x

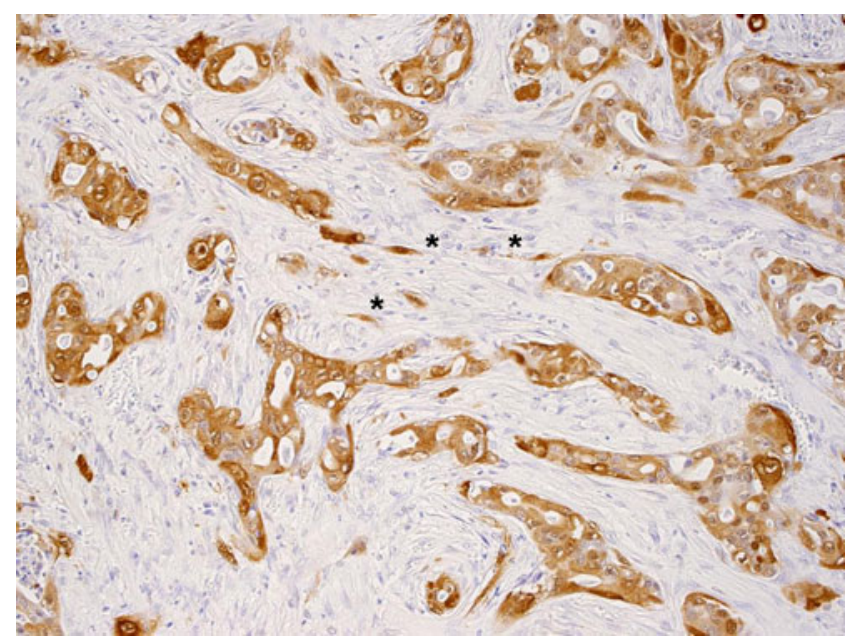

Fig. 3 MT antigen immunoreactivity in stromal fibroblasts marked with stars representing the membranous-cytoplasmic pattern of staining. Moderately differentiated adenocarcinoma; sample magnification $40 \mathrm{x}$

\section{Results}

The Immunoreactivity of Antigens in Adenocarcinomas and Their Stroma

\section{The Analysis of CD56 and CD57 Immunoreactivity in the Tissue Slides Examined}

CD56 antigen immunoreactivity was found in $14.2 \%$ of the adenocarcinoma samples and in $8 \%$ of the stromal samples (Fig. 1a). CD57 antigen immunoreactivity was demonstrated in $40 \%$ of the adenocarcinoma tissue slides and in $11.4 \%$ of

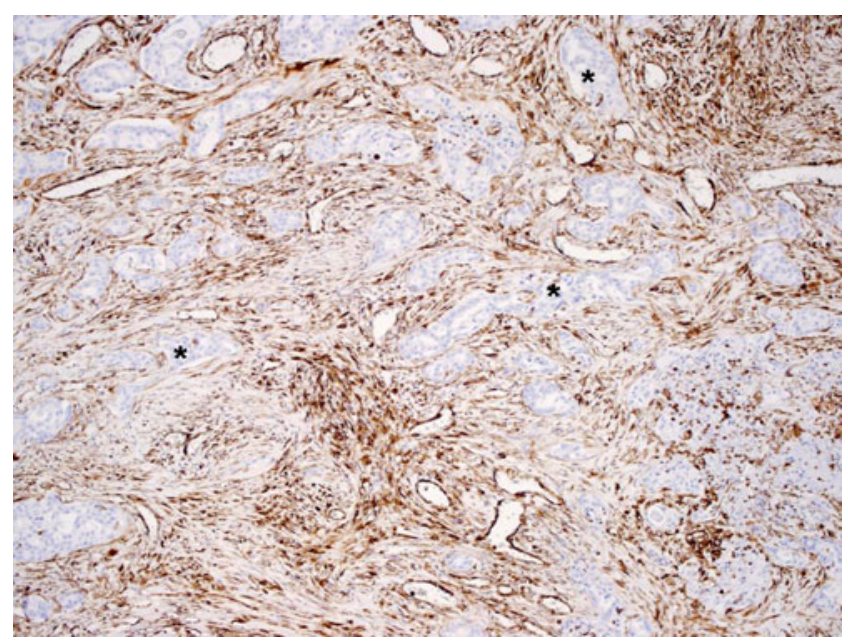

Fig. 4 Vimentin antigen immunoreactivity in adenocarcinoma and stromal tissue slides representing the membranous-cytoplasmic staining pattern. Tumor nests are almost vimentin negative (stars) while in the tumor microenvironment vimentin immunoreactivity is very strong and is represented by fibroblasts. Moderately differentiated adenocarcinoma; sample magnification $40 \times$ 


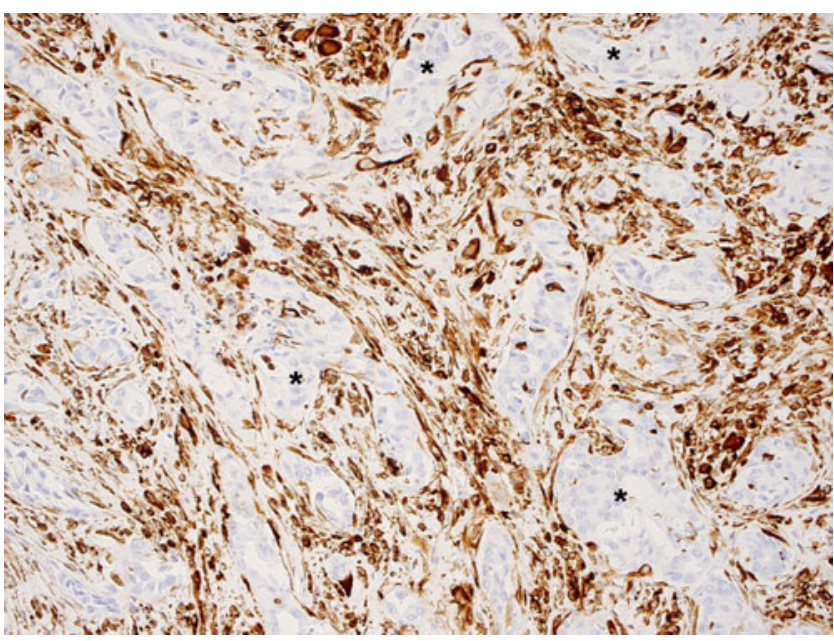

Fig. 5 Vimentin antigen immunoreactivity in adenocarcinoma and stromal tissue slides representing the membranous-cytoplasmic staining pattern. Tumor nests are almost vimentin negative (stars) while in the tumor microenvironment vimentin immunoreactivity is very strong and is represented by fibroblasts. Moderately differentiated adenocarcinoma; sample magnification $40 \times$

the stromal slides; it represented a cytoplasmic pattern of expression (Fig. 1b).

\section{The Analysis of MT Immunoreactivity in the Tissue Slides Examined}

MT antigen immunoreactivity was demonstrated in $68.5 \%$ of the adenocarcinoma tissue slides and most frequently exhibited the nuclear-cytoplasmic pattern of staining. The strongest MT immunoreactivity was observed in the marginal areas of the tumor nest, while in the center of the nest MT immunoreactivity was weaker (Fig. 2). MT immunoreactivity was also observed in $20 \%$ of the stromal tissue slides (Fig. 3) and represented the membranouscytoplasmic pattern of staining. This same immunoreactivity was also observed on stromal fibroblasts (Fig. 3).

\section{The Analysis of Vimentin Immunoreactivity in the Tissue Slides Examined}

Vimentin antigen immunoreactivity was demonstrated in $45.7 \%$ of the adenocarcinoma tissue slides and in $54.2 \%$ of the stromal tissue slides. The stromal fibroblasts strongly expressed vimentin and exhibited the membranous- cytoplasmic pattern of expression (Figs. 4 and 5). Vimentin immunoreactivity was not observed in the epithelia of the palatine tonsils.

\section{The Comparison of the Immunoreactivity Levels} of the Antigens Analyzed in Adenocarcinoma and Their Stromas

The results obtained for the immunoreactivity of the antigens analyzed in the tissue samples of adenocarcinomas and their stromas are presented in Table 3.

A statistically significantly higher number of CD57expressing cells were identified in the adenocarcinoma tissue slides than in the stromal slides. Also, a statistically significantly higher level of MT immunoreactivity was observed in the adenocarcinoma tissue slides than in the stromal slides. In turn, a statistically significantly higher vimentin immunoreactivity level was identified in the tumor microenvironment than in the tumor tissue slides.

The Comparison of Antigen Immunoreactivity Levels in the Adenocarcinoma and Reference Group Tissue Slides

The results obtained are presented in Table 4.

A statistically significantly higher number of CD56- and CD57-expressing cells were identified in the reference tissue slides than in the adenocarcinoma slides. In turn, a statistically significantly higher MT immunoreactivity level was identified in the adenocarcinoma tissue slides than in the reference tissue slides. There were no differences between the adenocarcinoma tissue slides and the reference slides as far as vimentin immunoreactivity.

The Comparison of the Levels of Antigen Immunoreactivity in the Stroma and Reference Group Tissues

The results obtained are presented in Table 5.

A statistically significantly higher number of CD56- and CD57-expressing cells were identified in the reference tissue slides than in the stromal slides. In turn, a statistically significantly higher level of vimentin immunoreactivity was identified in the tumor microenvironment than in the reference slides. There were no differences between the stroma and reference tissue slides as far as MT immunoreactivity
Table 3 The comparison of the immunoreactivity levels of the analyzed antigens in adenocarcinoma and the stroma

$I Q R$ the interquartile range; $N S$ non statistically significant;

\begin{tabular}{lccl}
\hline Antigen & Adenocarcinoma Median (IQR) & Stroma Median (IQR) & $p$ value \\
\hline CD56 & $0(0)$ & $0(0)$ & NS \\
CD57 & $0(2)$ & $0(0)$ & 0.01 \\
MT & $2(3)$ & $0(0)$ & 0.0002 \\
Vimentin & $0(0)$ & $3(1)$ & 0.004 \\
\hline
\end{tabular}


Table 4 The comparison of the immunoreactivity levels of the analyzed antigens in the adenocarcinoma and the reference group

$I Q R$ the interquartile range; $N S$ non statistically significant

\begin{tabular}{lccc}
\hline Antigen & Adenocarcinoma Median (IQR) & Reference group Median (IQR) & $p$ value \\
\hline CD56 & $0(0)$ & $1.5(2)$ & $p=0.01$ \\
CD57 & $0(2)$ & $2.4(3)$ & $p=0.01$ \\
MT & $2(3)$ & $0.1(0)$ & $P=0.001$ \\
Vimentin & $0(0)$ & $0(0)$ & NS \\
\hline
\end{tabular}

The analysis of antigen immunoreactivity with respect to tumor grade and size and the presence of lymph node metastases did not reveal any significant differences.

\section{Discussion}

In our present study the microenvironment of the adenocarcinoma was typified by the immunoreactivity of both vimentin and MT.

The remodeling of the tumor microenvironment would seem to involve the whole of the tissue adjacent to the tumor - the cells, the extracellular matrix, and the pattern of expressed proteins. The mesenchymal phenotype acquired by the tumor enables its local growth and dissemination and is typified by the expression of mesenchymal proteins such as vimentin $[9,10]$. In the present study we observed that the level of vimentin immunoreactivity was significantly higher in the tumor stroma than in either the tumor itself or the reference samples. This might be a consequence of the mesenchymal phenotype acquired by the tumor microenvironment as it seems to facilitate the progression of the tumor and the development of metastases. Additionally, we observed that any vimentin immunoreactivity in the tumor microenvironment was independent of the vimentin immunoreactivity found in the tumor itself. For example, there were tumors that did not express vimentin while their microenvironments did. Moreover, vimentin in the tumor microenvironment was expressed by the fibroblasts; this suggests that these fibroblasts represent carcinomaassociated fibroblasts, especially as vimentin is one of the markers used for the identification of these cells [18]. Overall, these findings suggest that the presence of vimentin immunoreactivity in the microenvironment of salivary gland adenocarcinoma could be an important marker of a remodeled microenvironment.
Next, we observed that metallothionein was expressed by both the tumor and the tumor stroma. The level was significantly higher in the tumor samples than in the stromal tissue samples, and significantly higher again in the tumor samples than in the reference samples. MT expression has been observed in various malignant tumors [22]. Within tumor nests MT has been mainly located in the peripheral part of tumor nests in cases of oral cavity squamous cell carcinoma. Such tumor cells were typified by a higher proliferative and lower apoptosis rate than the tumor cells located in the center of the nest [27,33]. Meanwhile, in nasopharyngeal cancer a negative correlation has been found between the MT expression and the rate of tumor cells undergoing apoptosis [33, 34]. Similarly, in head and neck squamous cell carcinoma, MT expression was found to be stronger in the peripheral portion of the tumor nests and its presence was identified in the adjacent stroma [24]. The type of MT immunoreactivity in the salivary gland adenocarcinoma in the present study was similar to the type of expression found in other tumors: it was the strongest in the peripheral parts of the tumor and weakest in the center of the tumor nests.

MT expression has also been observed in healthy tissues, such as in the basal part of normal epithelium in the oral cavity and nasopharynx. Its presence would seem to be linked to its pro-proliferative activity since this part of the epithelium is composed of the proliferating cells responsible for its renewal [27, 33, 34].

The presence of MT immunoreactivity in the stroma is an interesting phenomenon in itself. It has been demonstrated that MT can be expressed by healthy tissue in the tumor vicinity and its expression has been related to the presence of metastases in head and neck squamous cell carcinoma and in breast adenocarcinoma [24, 35]. MT expression in the tumor stroma has been speculated to result
Table 5 The comparison of the immunoreactivity levels of the analyzed antigens in the stroma and the reference group

$I Q R$ the interquartile range; $N S$ non statistically significant

\begin{tabular}{lccl}
\hline Antigen & Stroma Median (IQR) & Reference group Median (IQR) & $P$ value \\
\hline CD56 & $0(0)$ & $1.5(2)$ & $p=0.01$ \\
CD57 & $0(0)$ & $2.4(3)$ & $p=0.001$ \\
MT & $0(0)$ & $0.1(0)$ & NS \\
Vimentin & $3(1)$ & $0(0)$ & $p=0.001$ \\
\hline
\end{tabular}


from the increasing resistance to immune-mediated apoptosis $[24,35]$. In the present study we did not observe any significant relationship between the MT immunoreactivity and the presence of metastases. Interestingly, however, MT immunoreactivity in the tumor microenvironment was exhibited by stromal fibroblasts. Since these fibroblasts strongly expressed vimentin it would seem likely that they were cancer-associated fibroblasts [18] which are responsible for the modification of the tumor microenvironment as well as for determining tumor growth. The presence of cross-talk between the tumor cells and the stromal fibroblasts was confirmed in the co-cultured model of breast cancer. Additionally, it was shown that the fibroblasts from the tumor-stromal interface were the most active and were able to induce EMT and vimentin expression in a breast cancer model [36].

As MT expression protects the cell against apoptosis and promotes its proliferation, it would seem to be a marker of microenvironment remodeling. Moreover, MT- positive fibroblasts - that is, cancer-associated fibroblasts-would seem to form an integral part of the remodeled tumor microenvironment and also to condition tumor growth.

E. Canpolat et al. have postulated an important role for MT in immunomodulation. Since MT inhibits the immune response, it most likely helps to suppress the anticancer response [37]. In the light of MT's immunomodulating role, the presence of MT immunoreactivity in the tumor microenvironment may indicate that it is involved in creating the suppressive microenvironment.

The number of CD56- and CD57-expressing cells identified in both the adenocarcinoma and stroma tissue slides was significantly lower than in the reference group. There were no differences, however, in the number of CD56-expressing cells in the adenocarcinoma tissue slides in comparison to the stroma slides. In turn, a significantly higher number of CD57-expressing cells were identified in the adenocarcinoma than in the stroma tissue slides.

The level of anticancer response in the performed study, realized by CD56-expressing lymphocytes, seemed to remain at a comparable level in the tumor and its stroma, although the number of CD57-expressing lymphocytes was found to be higher in the tumor than in the stroma. Moreover, the number of effector cells in the tumor and its stroma remained significantly lower than in the control group; this indicated a deficit of these cells in the tumor and its microenvironment. Indeed, a decreased number of tumor-infiltrating lymphocytes in malignant tumors has been demonstrated in various neoplasms and correlated with tumor aggressiveness and poor prognosis $[38,39]$. It has also been determined that the function of TIL in malignant tumors is disturbed and that the decreased number of lymphocytes is accompanied by the alteration of their activity [40]. In gastric and colon cancers, the number of $\mathrm{T}$ CD56-expressing and $\mathrm{T}$ CD57-expressing lymphocytes was enhanced in the peripheral blood and in among the TIL [28, 29]. Additionally, the homeostasis between Th1/Th2 responses plays a key role in various types of immune responses, including the anticancer response [41]. The Th1 response has been shown in vivo in various malignant neoplasms to be fundamental in inducing a strong anticancer response [42]. In fact, in patients with advanced malignant tumors, the Th1 response is inhibited, while the Th2 response dominates [43].

In conclusion, the stroma of salivary gland adenocarcinoma is characterized by remodeling. The remodeling is represented by the expression of both vimentin and MT and by a deficit in CD57- and CD58-expressing cell infiltration. This situation would seem to be the result of immune tolerance for the tumor developing within the tumor microenvironment. Finally, the presence of MT and vimentin immunoreactivity in the fibroblasts of the tumor stroma may constitute a marker of active tissue remodeling.

Acknowledgements This work was supported by the State Committee for Scientific Research (KBN), Grant Number N403 032 31/2079, and also in part by the Jagiellonian University, Grant Number WL/ZKL/26/L.

Open Access This article is distributed under the terms of the Creative Commons Attribution Noncommercial License which permits any noncommercial use, distribution, and reproduction in any medium, provided the original author(s) and source are credited.

\section{References}

1. Witz IP (2009) The tumor microenvironment: the making of a paradigm. Cancer Microenvironment 2:S9-17

2. Scott AM, Wiseman G, Welt S, Adjei A, Lee FT, Hopkins W, Divgi CR, Hanson LH, Mitchell P, Gansen DN, Larson SM, Ingle JN, Hoffman EW, Tanswell P, Ritter G, Cohen LS, Bette P, Arvay L, Amelsberg A, Vlock D, Rettig WJ, Old LJ (2003) A Phase I dose-escalation study of sibrotuzumab in patients with advanced or metastatic fibroblast activation protein-positive cancer. Clin Cancer Res 9:1639-1647

3. Wesley UV, Albino AP, Tiwari S, Houghton AN (1999) A role for dipeptidyl peptidase IV in suppressing the malignant phenotype of melanocytic cells. J Exp Med 190:311-322

4. Dvorak HF (1986) Tumors: wounds that do not heal. Similarities between tumor stroma generation and wound healing. N Engl J Med 315:1650-1659

5. Lorusso G, Ruegg C (2008) The tumor microenvironment and its contribution to tumor evolution toward metastasis. Histochem Cell Biol 130:1091-1103

6. Acevedo VD, Gangula RD, Freeman KW, Li R, Zhang Y, Wang F, Ayala GE, Peterson LE, Ittmann M, Spencer DM (2007) Inducible FGFR-1 activation leads to irreversible prostate adenocarcinoma and an epithelial-to-mesenchymal transition. Cancer Cell 12:559-571

7. Hugo H, Ackland ML, Blick T, Lawrence MG, Clements JA, Williams ED, Thompson EW (2007) Epithelial-mesenchymal and mesenchymal-epithelial transitions in carcinoma progression. J Cell Physiol 213:374-383

8. Xu J, Wang R, Xie ZH, Odero-Marah V, Pathak S, Multani A, Chung LW, Zhau HE (2006) Prostate cancer metastasis: role of the 
host microenvironment in promoting epithelial to mesenchymal transition and increased bone and adrenal gland metastasis. Prostate 66:1664-1673

9. Grunert S, Jechlinger M, Beug H (2003) Diverse cellular and molecular mechanisms contribute to epithelial plasticity and metastasis. Nat Rev Mol Cell Biol 4:657-665

10. Kokkinos MI, Wafai R, Wong MK, Newgreen DF, Thompson EW, Waltham M (2007) Vimentin and epithelial-mesenchymal transition in human breast cancer-observations in vitro and in vivo. Cells Tissues Organs 185:191-203

11. Thiery JP (2002) Epithelial-mesenchymal transitions in tumour progression. Nat Rev Cancer 2:442-454

12. Paccione RJ, Miyazaki H, Patel V, Waseem A, Gutkind JS, Zehner ZE, Yeudall WA (2008) Keratin down-regulation in vimentinpositive cancer cells is reversible by vimentin RNA interference, which inhibits growth and motility. Mol Cancer Ther 7:2894-2903

13. Powell DW, Mifflin RC, Valentich JD, Crowe SE, Saada JI, West AB (1999) Myofibroblasts. I. Paracrine cells important in health and disease. Am J Physiol 277:C1-C9

14. Vered M, Allon I, Buchner A, Dayan D (2009) Stromal myofibroblasts accompany modifications in the epithelial phenotype of tongue dysplastic and malignant lesions. Cancer Microenvironment 2:49-57

15. De Wever O, Mareel M (2005) Role of tissue stroma in cancer cell invasion. J Pathol 200:429-447

16. Hayward SW, Wang Y, Cao M, Hom YK, Zhang B, Grossfeld GD, Sudilovsky D, Cunha GR (2001) (2001) Malignant transformation in a nontumorigenic human prostatic epithelial cell line. Cancer Res 61:8135-8142

17. Orimo A, Gupta PB, Sgroi DC, Arenzana-Seisdedos F, Delaunay T, Naeem R, Carey VJ, Richardson AL, Weinberg RA (2005) Stromal fibroblasts present in invasive human breast carcinomas promote tumor growth and angiogenesis through elevated SDF-1/ CXCL12 secretion. Cell 121:335-348

18. Sugimoto H, Mundel TM, Kieran MW, Kalluri R (2006) Identification of fibroblast heterogeneity in the tumor microenvironment. Cancer Biol Ther 5:1640-1646

19. Klaassen CD, Liu J, Choudhuri S (1999) Metallothionein: an intracellular protein to protect against cadmium toxicity. Annu Rev Pharmacol Toxicol 39:267-294

20. Apostolova MD, Cherian MG (2000) Nuclear localization of metalothionein during cell proliferation and differentiation. Cell Mol Biol 46:347-356

21. Theocharis SE, Margeli AP, Klijanienko JT, Kouraklis GP (2004) Metallothionein expression in human neoplasia. Histopathology 45:103-18

22. Cherian MG, Jayasurya A, Bay BH (2003) Metallothioneins in human tumors and potential roles in carcinogenesis. Mutat Res 33:201-209

23. Fan LZ, Cherian MG (2002) Potential role of p53 on metallothionein induction in human epithelial breast cancer cells. Br J Cancer 87:1019-1026

24. Dutsch-Wicherek M, Popiela TJ, Klimek M, Rudnicka-Sosin L, Wicherek L, Oudinet JP, Skladzien J, Tomaszewska R (2005) Metallothionein stroma reaction in tumor adjacent healthy tissue in head and neck squamous cell carcinoma and breast adenocarcinoma. NeuroEndocrinol Lett 26:567-574

25. Dutsch-Wicherek M, Sikora J, Tomaszewska R (2008) The possible biological role of metallothionein in apoptosis. Front Biosci 13:4029-4038

26. Hellquist HB (1997) Apoptosis in epithelial hyperplastic laryngeal lesions. Acta Otolaryngol Suppl 527:25-29
27. Sundelin K, Jadner M, Norberg-Spaak L, Davidsson A, Hellquist HB (1997) Metallothionein and Fas (CD95) are expressed in squamous cell carcinoma of the tongue. Eur J Cancer 33:1860-1864

28. Okada T, Iiai T, Kawachi Y, Moroda T, Takii Y, Hatakeyama K, Abo T (1995) Origin of CD57+ T cells which increase at tumour sites in patients with colorectal cancer. Clin Exp Immunol 102:159-166

29. Takii Y, Hashimoto S, Iiai T, Watanabe H, Hatakeyama K, Abo T (1994) Increase of the proportion of granulated CD56+ T cells in patients with malignancy. J Clin Exp Immunol 97:522-527

30. Satoh M, Seki S, Hashimoto W, Ogasawara K, Kobayashi T, Kumagai K, Matsuno S, Takeda K (1996) Cytotoxic gammadelta or alphabeta $\mathrm{T}$ cells with a natural killer cell marker, CD56, induced from human peripheral blood lymphocytes by a combination of IL-12 and IL-2. J Immunol 157:3886-3892

31. Kuss I, Hathaway B, Ferris RL, Gooding W, Whiteside TL (2004) Decreased absolute counts of $\mathrm{T}$ lymphocyte subsets and their relation to disease in squamous cell carcinoma of the head and neck. Clin Cancer Res 10:3755-3762

32. Sallusto F, Geminat J, Lanzavecchia A (2004) Central memory and effector memory $\mathrm{T}$ cell subsets: function, generation, and maintenance. Annu Rev Immunol 22:745-763

33. Jayasurya A, Bay BH, Yap WM, Tan NG (2000) Correlation of metallothionein expression with apoptosis in nasopharyngeal carcinoma. Br J Cancer 82:1198-1203

34. Jayasurya A, Bay BH, Yap WM, Tan NG (2000) Infiltrating lymphocytes in undifferentiated nasopharyngeal cancer lack metallothionein expression. Cancer Lett 155:99-104

35. Popiela TJ, Rudnicka-Sosin L, Dutsch-Wicherek M, Klimek M, Basta P, Galazka K, Wicherek L (2006) The metallothionein and RCAS1 expression analysis in breast cancer and adjacent tissue regarding the immune cells presence and their activity. NeuroEndocrinol Lett 27:786-794

36. Gao MQ, Kim BG, Kang S, Choi YP, Park H, Kang KS, Cho $\mathrm{NH}$ (2010) Stromal fibroblasts from the interface zone of human breast carcinomas induce an epithelial-mesenchymal transition-like state in breast cancer cells in vitro. J Cell Sci 123:3507-3514

37. Canpolat E, Lynes MA (2001) In vivo manipulation of endogenous metallothionein with a monoclonal antibody enhances a $\mathrm{T}$ dependent humoral immune response. Toxicol Sci 62:61-70

38. Ioachim HL (1979) The stroma reaction of tumors: An expression of immune surveillance. J Cell Biochem Suppl 57:465-475

39. Keller SE, Ioachim HL, Pearse T, Siletti DM (1976) Decreased Tlymphocytes in patients with mammary cancer. Am J Clin Pathol 65:445-449

40. Miescher S, Whiteside TL, Moretta L, Von Fliedner V (1987) Clonal and frequency analyses of tumor infiltrating $\mathrm{T}$ lymphocytes from human solid tumors. J Immunol 138:4004-4011

41. Mosmann TR, Cherwinski H, Bond MW, Giedlin MA, Coffman RL (1986) Two types of murine helper T cell clone. I. Definition according to profiles of lymphokine activities and secreted proteins. J Immunol 136:2348-2357

42. Trinchieri G (1995) Interleukin-12- a cytokine produced by antigen-presenting cells with immunoregulatory functions in the generation of T-helper cell type 1 and cytotoxic lymphocytes. Blood 84:4008-4027

43. Elsässer-Beile U, Kölble N, Grussenmeyer T, Schultze-Seemann W, Wetterauer U, Gallati H, Schulte Mönting J, von Kleist S (1998) Th1 and Th2 cytokine response patterns in leukocyte cultures of patients with urinary bladder, renal cell and prostate carcinomas. Tumor Biol 19:470-476 